\author{
Neurology \\ Issue: Volume 50(2), February 1998, pp 368-373 \\ Copyright: (C) 1998 American Academy of Neurology \\ Publication Type: [Articles] \\ DOI: 10.1212/WNL.50.2.368 \\ ISSN: 0028-3878 \\ Accession: 00006114-199802000-00017
}

[Articles]

\title{
Cognitive function in nondemented older women who took estrogen after menopause
}

Jacobs, D. M. PhD; Tang, M.-X. PhD; Stern, Y. PhD; Sano, M. PhD; Marder, K. MD; Bell, K. L. MD; Schofield, P. MD; Dooneief, G. MD; Gurland, B. MD; Mayeux, R. MD, MSE

\section{Author Information}

From the Gertrude H. Sergievsky Center (Drs. Jacobs, Tang, Stern, Sano, Marder, Bell, Schofield, Dooneief, and Mayeux), the Departments of Neurology(Drs. Jacobs, Tang, Stern, Sano, Marder, Bell, Schofield, Dooneief, and Mayeux) and Psychiatry (Drs. Stern, Gurland, and Mayeux), the Taub Center for Alzheimer's Disease Research (Drs. Jacobs, Stern, Sano, Marder, Bell, Dooneief, and Mayeux), the Stroud Center for the Study of Quality of Life(Dr. Gurland), and the Division of Epidemiology (School of Public Health)(Dr. Mayeux), Columbia University College of Physicians and Surgeons, New York, NY.

Supported by Federal Grants AG07232, AG10963, AG08702, and RR00645 and by the Charles S. Robertson Memorial Gift for Alzheimer's Disease Research from the Banbury Fund. Dr. Tang is supported by a Faculty Scholar Award (95-045) from the Alzheimer's Disease and Related Disorders Association.

Received February 19, 1997. Accepted in final form September 19, 1997.

Address correspondence and reprint requests to Dr. Diane M. Jacobs, Gertrude H. Sergievsky Center, 630 West 168 Street, New York, NY 10032.

\section{Article abstract}

Investigations of the effects of estrogen replacement on cognitive function in healthy older women have yielded disparate results. We evaluated the relationship between a history of estrogen use and cognitive test performance in 727 women participating in a large community-based study. Participants were followed longitudinally for an average of 2.5 years. Estrogen use history was obtained at baseline. Standardized tests of memory, language, and abstract reasoning were administered at baseline and at follow-up. Results indicate that women who had used estrogen replacement scored significantly higher on cognitive testing at baseline than nonusers, and their performance on verbal memory improved slightly over time. The effect of estrogen on cognition was independent of age, education, ethnicity, and APOE genotype. Results suggest that estrogen replacement therapy may help to maintain cognitive function in nondemented postmenopausal women.

Recent evidence suggests that estrogen replacement therapy in postmenopausal women may help to maintain cognitive function in old age. Beneficial effects associated with estrogen use in older women have been observed on tests of immediate and delayed paragraph recall 1 and proper name recall.2 Estrogen replacement also has been associated with a decreased risk for Alzheimer's disease 3-5 and with enhanced responsiveness to treatment with cholinergic agonists in patients with Alzheimer's disease.6 
Although results from investigations of volunteer and patient-based samples have suggested that estrogen use has a positive effect on cognition, population-based studies of estrogen and cognitive function in older women have yielded inconsistent results. A report from the Baltimore Longitudinal Study of Aging indicates that estrogen replacement therapy was associated with better performance on the Benton Visual Retention Test, a measure of nonverbal memory.7 Estrogen users in the Rancho Bernardo cohort scored higher than nonusers on Category Fluency and the Mini-Mental State Examination; however, given the large number of statistical comparisons performed in this report, the authors concluded that there was"no consistent evidence for an effect of estrogen on cognitive function."8 Differences between studies in the characteristics of participants and the neuropsychological measures used to assess cognitive function may have contributed to the contradictory results.

We recently reported that women participating in a community-based epidemiologic study of aging and dementia in northern Manhattan, New York who had used estrogen during the postmenopausal period had a decreased risk of developing Alzheimer's disease. 5 In the current study, we examined the association between estrogen use and cognitive function as assessed by standardized neuropsychological measures in nondemented women from this same cohort. In addition to performing cross-sectional comparisons of estrogen users and nonusers, we examined the effect of estrogen use on longitudinal change in performance on tests of cognitive function.

Methods. Study population. Data were obtained from women participating in a community-based epidemiologic study of aging and dementia in northern Manhattan, New York. Participants included a random sample of Medicare recipients in a geographically defined region and elderly volunteers from senior centers and elder housing sites in the same community. The population from which participants were drawn is ethnically diverse and is comprised primarily of African-American, Hispanic, and white elders. Participation of eligible subjects in the epidemiologic study represented $77 \%$ of individuals at the senior centers or housing sites and $72 \%$ of the Medicare sample. Participation from either source did not differ by sex or ethnic group.

We identified 883 women who were free of dementia or questionable dementia(i.e., Clinical Dementia Rating of $0.5) 9$ at their initial evaluation. Of these, 727 women met all criteria for inclusion in the current analyses; 415 women were from the random sample survey, and 312 were recruited from community senior centers. Potential participants were excluded if they had signs of stroke $(n=28)$ or Parkinson's disease $(n=73)$ or if they had incomplete data on the neuropsychological measures $(n=34)$ or estrogen-use questionnaire $(n=34)$ (some women met more than one exclusion criterion).

Procedures. Participants were evaluated by a physician who performed a standardized physical and neurologic examination and recorded each subject's medical history. Participants also were given a brief neuropsychological test battery that consisted primarily of subtests and short forms of widely used neuropsychological measures. 10 Medical, neuropsychological, and all available ancillary information, including medical records and imaging studies, were used in the evaluation.

We examined the association between estrogen use and performance on four measures from three tests in our battery. These measures were chosen a priori based on previous research that has shown them to be highly sensitive to subtle cognitive change.11-13 The Selective Reminding Test 14 is a measure of verbal recall memory. Participants were given six trials to learn a list of 12 unrelated words. After each recall attempt, a selective reminding procedure was used wherein participants were reminded only of those words that had not been successfully recalled. To assess long-term retention of the word list, a single delayed-recall trial was administered after a 15-minute interval. The Similarities subtest of the Wechsler Adult Intelligence Scale-Revised(WAIS-R)15 is a test of verbal abstract reasoning and concept formation. Participants are required to provide relevant similarities or superordinate categories for paired items. A 15-item version of the Boston Naming Test 16 was used to assess word-finding ability. This test of visual confrontation naming requires participants to identify line drawings of objects. 
History of estrogen use was obtained as part of a risk factor questionnaire given to all women at the initial assessment. The test-retest reliability of the questionnaire over a 12-month interval was established previously 17: the reliability of questions related to the use of estrogen was found to be in the fair to good range 18 (Kappa $=0.65$ ). Women were asked to identify the estrogen preparation used, but dosages were not queried.

Data analysis. Age, education, and ethnicity were treated as covariates for all statistical analyses, which were performed using SPSS for Windows (version 6.0, SPSS Inc., Chicago, IL).

To assess the effect of estrogen use on cognitive test performance cross-sectionally, we performed two MANCOVAs. This statistic examines group differences on all dependent variables (i.e., neuropsychological test scores) simultaneously, thereby reducing the risk of a false-positive result that is associated with performing a series of univariate analyses of covariance. For both analyses, baseline scores from the measures of cognition were included as dependent variables. Scores examined were total immediate recall (summed across the six acquisition trials; maximum, 72) and delayed recall(maximum, 12) on the Selective Reminding Test, WAIS-R Similarities scaled score (maximum, 19), and total correct on the Boston Naming Test (maximum, 15). The first MANCOVA compared women who had never taken estrogen replacement (never-users) to those who had taken estrogen at any time for any duration during the postmenopausal period (ever-users). (The number of current estrogen users in our cohort was insufficient to examine them as a separate group; therefore, current and past estrogen users were combined into ever-users.) For the second MANCOVA, we dichotomized ever-users into two groups based on the median duration of estrogen use, thereby yielding a three-group analysis: women who had never taken estrogen, women who had taken estrogen replacement for less than the median duration, and women who had taken estrogen for longer than the median duration. Post-hoc univariate ANCOVAs were performed to determine which neuropsychological measures contributed to significant multivariate results.

To determine whether group differences in cognition were associated with differences in mood-related symptoms, we also compared the scores of estrogen users and nonusers on the Hamilton Rating Scale for Depression using ANCOVA. The Hamilton Rating Scale was administered to a subset of participants during the baseline evaluation; limitations during the early phases of the study in personnel trained in the standard research administration of the Hamilton Rating Scale precluded the administration of this measure to all participants. The decision as to whether or not a Hamilton was administered was based on tester availability and was independent of estrogen use history or other subject characteristics.

Finally, longitudinal change on each of the neuropsychological measures from initial to follow-up testing was assessed using repeated measures ANCOVAs.

Secondary analyses. To determine whether participants recruited from senior centers and those from the community-based random sample differed in terms of estrogen use history or the effect of estrogen on cognition, all analyses were repeated separately for these two subject groups.

Because current estrogen users were included in our group of ever-users, it was possible that an acute effect of estrogen on cognition, if present, could influence our results by raising the group average scores for ever-users. To eliminate this possibility, all analyses were repeated after excluding current users, thereby comparing past-users to never-users.

Finally, the inclusion of participants in the incipient stages of dementia also could bias our results, particularly because estrogen use has been associated with a decreased risk for Alzheimer's disease.3-5 As described earlier, all included women were free of dementia or questionable dementia at their initial assessment. In an effort to further minimize the influence of incipient dementia on our findings, analyses were repeated after excluding women who became demented during the follow-up period. 
Results. The average age of the 727 women was 74.2 years (SD, 6.9 years), and participants had completed an average of 9.4 years of education (SD, 4.6 years). The sample was $27.5 \%$ white, $27.5 \%$ African-American, $43.7 \%$ Hispanic, and $1.4 \%$ other race/ethnicity. Eighty-one women (11\%) reported taking estrogen after menopause. The average duration of estrogen use was $4.55+/-8.64$ years, and the median was 1 year; 37 women ( $5 \%$ of the total sample) had used estrogen for longer than 1 year (mean, 9.83 years), whereas 44 ( $6 \%$ of the total sample) had used estrogen for a year or less (mean, 2.72 months). Only 12 women ( $2 \%$ of the total sample) were still using estrogen at the time of evaluation. Most women received estrogen therapy around the time of menopause. The average interval between last use of estrogen and cognitive evaluation in women with a previous history of estrogen use was $24.5+/-11.8$ years. There was no significant association between when a woman last used estrogen and performance on measures of cognition after covarying for age at the time of assessment $(r=-0.19$ to $0.09 ; p=0.12$ to 0.45 ). The most common estrogen preparation used was unopposed oral estrogen(Premarin, Wyeth-Ayerst Laboratories, Philadelphia, PA); information on dosage was not queried and therefore is unavailable.

Characteristics of estrogen users and nonusers are provided in table 1. Subject groups were of comparable age at the time of testing $(p>0.10)$. Never-users had completed fewer years of formal education than ever-users in general and women who had used estrogen for longer than 1 year in particular. Fewer African-American and Hispanic women had used estrogen than white women (African-American 7.5\%, Hispanic 8.8\%, white 19\%; $p<$ 0.0004). Results of apolipoprotein E (apoE) genotyping were available for $49 \%$ of participants ( $49 \%$ of never-users and $52 \%$ of ever-users). The distribution of apoE genotype among ever-users and never-users was comparable $\left([c h i]^{2}=0.46 ; p=0.80\right)$. Prevalence of medical conditions potentially associated with estrogen use and cognitive function, specifically myocardial infarction, congestive heart failure, hypertension, hypercholesterolemia, atrial fibrillation, or other cardiac arrhythmia, also did not differ across groups(data not shown; all $p>0.10$ ).

\begin{tabular}{|c|c|c|c|c|}
\hline & Never used estrogen & Ever used estrogen & Used estrogen $\leq 1 \mathrm{y}$ & Used estrogen $>1 y$ \\
\hline Number of women & 646 & 81 & 44 & 37 \\
\hline Age at initial evaluation (y) & $74.3=6.8$ & $73.8 \div 7.4$ & $75.2 \pm 7.2$ & $72.2 \pm 7.4$ \\
\hline Education* $(y)$ & $9.1 \pm 4.5$ & $11.0 \div 4.5 \dagger$ & $10.0 \pm 4.5$ & $12.2 \pm 4.2 \dagger$ \\
\hline \multicolumn{5}{|l|}{ Apolipoprotein E genotypeł } \\
\hline$\epsilon 2 / \epsilon 2, \epsilon 2 / \epsilon 3$ & $10.8(34)$ & $14.3(6)$ & $19.4(6)$ & $0(0)$ \\
\hline$\epsilon 4 / \epsilon 4, \epsilon 4 / \epsilon 3$ & $25.1(79)$ & $23.8(10)$ & $25.8(8)$ & $18.2(2)$ \\
\hline$e 3 / 63$ & $64.1(202)$ & $61.9(26)$ & $54.8(17)$ & $81.8(9)$ \\
\hline
\end{tabular}

Table 1 Characteristics of subject groups

Results of the MANCOVA comparing estrogen ever-users and never-users while statistically controlling for the effects of age, education, and ethnicity yielded a significant result $(F=5.04, p=0.001)$. Post-hoc univariate ANCOVAs revealed significant group differences on all four neuropsychological measures included in the multivariate analysis (table 2). The effects of estrogen were independent of apoE genotype: within each apoE genotype group, ever-users scored higher than never-users on every neuropsychological measure.

\begin{tabular}{|c|c|c|c|c|}
\hline & Never used estrogen & Ever used estrogen & Used estrogen $\leq 1 \mathrm{y}$ & Used estrogen $>1 y$ \\
\hline \multicolumn{5}{|l|}{ SRT } \\
\hline Immediate recall & $39.8=8.5$ & $44.0 \pm 8.8^{\circ}$ & $42.6=8.9 \dagger$ & $45.6 \pm 8.5 \neq \xi$ \\
\hline Delayed recall & $5.8=2.3$ & $6.7 \pm 2.5 t$ & $6.4 \pm 2.5$ & $7.2 \pm 2.5+1$ \\
\hline Similarities Scaled Score & $5.8=2.8$ & $7.6 \pm 3.46^{*}$ & $6.9 \pm 3.2 \dagger$ & $8.4 \pm 3.4^{*} \xi$ \\
\hline Boston Naming Test (15-item) & $13.5 \pm 1.6$ & $14.1 \pm 1.2 \dagger$ & $14.0 \pm 1.3 \dagger$ & $14.2 \pm 1.0+\xi$ \\
\hline
\end{tabular}

Comparison with never-users using ANCOVA controlling for age, education, and ethnicity: ${ }^{*} p<0.01,+p<0.05, \neq p<0.10$. Comparison with a demographically matched group of never-users using ANOVA: $\$ p<0.05, \| p<0.1$.

$\mathrm{SRT}=$ Selective Reminding Test.

Table 2 Mean +/- SD scores on tests of cognition 
Data on the Hamilton Rating Scale for Depression were available for $63 \%$ of participants $(62 \%$ never-users, $75 \%$ ever-users). Level of depression did not differ by estrogen use history (ever-users, mean $=4.85, \mathrm{SD}=4.53$; neverusers, mean $=4.03, \mathrm{SD}=4.11 ; p=0.28$ ).

The three-group MANCOVA comparing women who had never taken estrogen to women who had taken estrogen for 1 year or less and those who had taken estrogen for longer than 1 year also yielded a significant result $(F=$ $2.56, p=0.009$ ). Women who had never taken estrogen scored significantly lower than women who had taken estrogen for less than 1 year on all four neuropsychologic measures and significantly lower than women who had taken estrogen for more than 1 year on WAIS-R Similarities (see table 2). Comparisons of never-users to women who had taken estrogen for longer than 1 year on measures of memory and language approached significance $(p<$ 0.10). Although women who had used estrogen for 1 year or less consistently scored lower than those who had taken estrogen for more than 1 year, these comparisons were not statistically significant.

To more directly compare women who had used estrogen for more than 1 year to those who had never used estrogen, we repeated the comparisons after matching the groups on the demographic covariates. Stratified random sampling was used to create a group of never-users who were comparable with women who had used estrogen for more than 1 year in terms of age (mean $=73.39$ years), education (mean $=11.99$ years), and ethnicity $(60 \%$ white, 16\% African-American, 24\% Hispanic). One-way ANOVAs of neuropsychological measures using these two demographically matched groups yielded significant group differences for immediate recall on the Selective Reminding Test ( $F=4.09, p=0.04)$, WAIS-R Similarities ( $F=5.16, p=0.02)$, and Boston Naming $(F=4.51, p=$ $0.03)$ and a trend toward significance for delayed recall on the Selective Reminding Test $(F=3.34 ; p=0.07)$.

Data on follow-up neuropsychologic testing were available for 522 women. Women with and without follow-up data did not differ in age, education, ethnicity, or estrogen use history. The average interim between initial and follow-up testing was $2.55+/-1.38$ years for never-users and $2.75+/-1.31$ years for ever-users. Repeated measures ANCOVAs revealed a significant interaction of group (ever-users versus never-users) by time(baseline - follow-up testing) for immediate $(F=6.61, p=0.01)$ and delayed recall $(F=12.73, p<0.001)$ on the Selective Reminding Test. On both measures, scores of ever-users improved on follow-up testing, whereas the scores of never-users declined (immediate recall, ever-users +1.58 , never-users -1.32 ; delayed recall, ever-users +0.64 , never-users -0.41). There was a significant main effect of group on WAIS-R Similarities $(F=15.82, p<0.0001)$ and Boston Naming Test $(F=6.72, p=0.01)$ but no significant effect of time or a group-by-time interaction.

Results of secondary analyses. The random sample of Medicare recipients and senior center volunteer cohorts were comparable in terms of percentage of estrogen users $(10.6 \%$ and $11.9 \%$, respectively), and multivariate comparisons of estrogen ever-users and never-users were significant in each cohort when analyzed separately $(p<$ 0.04). The multivariate comparison of never-users with past-users who had used estrogen for greater than and less than 1 year was significant in the random sample cohort $(F=2.01 ; p=0.05)$ and approached significance in the senior center volunteer cohort $(F=1.77 ; p=0.08)$.

Current estrogen users were significantly younger at the time of evaluation (mean $=66.75, \mathrm{SD}=6.36$ ) and better educated (mean $=14.75 ; \mathrm{SD}=3.60$ ) than past-users and never-users; therefore, excluding current users from our group of ever-users resulted in a somewhat closer match between estrogen users and nonusers on these demographic variables (mean age, 74.29 for never-users and 75.07 for past-users, $p=0.37$; mean education, 9.15 for never-users and 10.35 for past-users, $p=0.03$ ). Excluding current-users from the analyses had no effect, however, on results of group comparisons on the cognitive tests: MANCOVAs comparing never-users with pastusers $(F=3.97 ; p=0.003)$ and never-users with past-users who had used estrogen for greater than and less than 1 year $(F=2.02 ; p=0.04)$ remained significant. Specific tests contributing to the significant multivariate results were identical to the previous set of analyses (data not shown). The outcomes of repeated-measures comparisons of longitudinal change in cognition also were unchanged after excluding current users; significant group-by-time interactions again were obtained on measures of immediate $(F=5.85 ; p=0.02)$ and delayed $(F=10.93 ; p=0.001)$ verbal memory, with past-users improving slightly on these measures and never-users declining slightly. 
Thirty-three women were diagnosed as demented at the follow-up evaluation. Thirty-two of these women had never used estrogen and 1 had used estrogen for less than 1 year. None of the women who had used estrogen for more than 1 year became demented during the follow-up period. Results of our analyses of cognitive function, however, were unchanged after excluding participants who subsequently became demented; multivariate comparisons remained significant when comparing never-users to ever-users overall $(F=4.58 ; p=0.001)$ and after splitting everusers into those who had taken estrogen for more or less than 1 year $(F=2.35 ; p=0.02)$. Of particular note, even after excluding participants who became demented, never-users declined slightly from the initial to follow-up examination on measures of verbal memory, whereas ever-users improved ( $p=0.04$ for SRT immediate recall and $p=0.001$ for delayed recall).

Discussion. In a multiethnic community-based cohort of nondemented older women, we found that a history of estrogen use during the postmenopausal period was associated with higher scores on neuropsychological measures of memory, abstract reasoning, and language. Further, longitudinal change in memory test scores over 2 years was significantly different for ever-users and never-users of estrogen. Scores increased slightly for ever-users but decreased slightly for never-users. Estrogen ever-users and never-users did not differ in prevalence of cardiovascular disease or symptoms of depression nor in the distribution of apoE genotype; therefore, these potentially confounding factors do not account for the current findings.

Results of this study are consistent with several previous reports showing the benefits of estrogen use on cognitive function in older women.1,2,7,19,20 Group comparisons on specific measures of cognition, however, have differed somewhat across studies. Previous reports suggest that estrogen maintains verbal 1,19,20 or visual 7 memory in older women but may have no effect on other cognitive functions. The current results, however, suggest that the effects of estrogen are not limited to the memory domain but include abstract reasoning and language as well.

No consistent effect of estrogen use on cognition was found by Barrett-Connor and Kritz-Silverstein in the Rancho Bernardo cohort.8 Differences between the cohorts of women in their study and ours may have contributed to the disparate results. First, we excluded from our analyses all women with signs of dementia, stroke, and Parkinson's disease, whereas Barrett-Connor and Kritz-Silverstein may not have excluded these individuals. The inclusion of participants with conditions that are known to affect performance on tests of cognition may have influenced some of their results. The two cohorts also differ markedly in terms of the percentage of women with a positive history of estrogen use. Eleven percent of our cohort were ever-users, and only $2 \%$ were currently using estrogen at the time of the initial evaluation. In contrast, $73.5 \%$ of the women in the study reported by Barrett-Connor and KritzSilverstein had used estrogen at some time postmenopausally and $33.5 \%$ were current users. Our cohort is ethnically diverse and has relatively limited educational attainment, whereas the Rancho Bernardo cohort is almost exclusively white and most of the women had completed some college education; however, it is unclear why estrogen would differentially affect women of different ethnicity or educational attainment. Finally, because both studies were observational rather than randomized clinical trials, we cannot exclude the possibility that unmeasured subject characteristics were associated with estrogen use and that these differences influenced the observed results. 
Estrogen may affect cognition in postmenopausal women via effects on cholinergic and nerve growth factor (NGF)related systems in the brain. Estrogen affects the expression of choline acetyltransferase and NGF receptors in cholinergic neurons of the basal forebrain 21 and increases the density of dendritic spines 22 and the synthesis of acetylcholine transferase 23 in area CA1 of the hippocampus. Miranda et al.24 reported co-localization of estrogen target neurons and mRNAs for neurotrophins and their receptors in the hippocampus, basal forebrain, and cerebral cortex. Postmenopausal estrogen replacement therapy may help to preserve the integrity of cholinergic activity in the hippocampus, basal forebrain, and other structures known to be vital to maintenance of normal cognitive, particularly mnemonic, function. Although our results indicate that longer exposures to postmenopausal estrogen therapy are associated with relatively higher scores on measures of cognition, women with very brief exposures also performed better than women with no history of estrogen use. Similar results have been reported in studies examining the effect of estrogen replacement therapy on incidence of Alzheimer's disease. It appears that administration of exogenous estrogen, even for a limited period of time, may delay or temporarily halt neuronal changes associated with estrogen deficiency.

All participants were free of dementia or questionable dementia at the initial evaluation; however, the inclusion of women in the very early or preclinical phase of dementia could have contributed to the observed results. The measures of cognition used in the current study have been shown to be sensitive to the subtle cognitive changes associated with preclinical Alzheimer's disease.11-13 In an effort to evaluate the potential contribution of the inclusion of women with very mild dementia, we repeated all analyses after excluding participants who became demented between the initial and follow-up examination, and results were unchanged. It appears, therefore, that estrogen may affect cognition in older women independent of its effect on the incidence of Alzheimer's disease. Nevertheless, the possibility remains that the relatively lower and declining scores of women who had never used estrogen were associated with the early stages of a progressive dementia in these women but that their cognitive impairment was too mild to impair their function at the time of our assessments. We are continuing to follow this cohort and will be able to confirm whether or not our non-demented participants remain free of dementia.

Because our findings are from an observational study, there are some limitations in the conclusions that can be drawn from them. Although we statistically controlled for the effects of several important demographic characteristics (i.e., age, education, and ethnicity) in our analyses, other unmeasured subject characteristics or factors may have contributed to our results. For example, access to health care or ability to pay for medications may have influenced the association between estrogen use and cognitive function. Similarly, it is possible that women who were more cognitively intact or those who were well educated were more likely to have used estrogen replacement because they exerted greater initiative in seeking medical care at the time of menopause.

Nevertheless, the finding of longitudinal and cross-sectional differences in test performance between estrogen users and nonusers suggests that pre-existing cohort differences probably did not account entirely for the observed results. As with all retrospective studies, recall bias is a potentially confounding factor; women with relatively better cognitive functioning may have been more likely to remember having used estrogen replacement in the past. Finally, no data on the dosage of estrogen replacement were available; hence, we were unable to assess the effects of this potentially important variable. A prospective clinical trial is needed to confirm our findings of the effects of estrogen replacement on cognitive function in postmenopausal women and to clarify issues regarding the safety and efficacy of dosages and durations of treatment.

\section{Acknowledgments}

We thank Drs. David Wilder and Rafael Lantigua from the Stroud Center for the Study of Quality of Life and the Center for Geriatrics and Gerontology at Columbia University for their efforts in the design and execution of the registry and Medicare survey. We are grateful to Rosann Costa, Maria Gonzales, and the interview team from the Sergievsky Center and the Center for Geriatrics and Gerontology at Columbia University for their assistance with this project. We also thank Dr. Dale Hesdorffer for her helpful comments on an earlier version of this article.

\section{References}

1. Kampen DL, Sherwin BB. Estrogen use and verbal memory in healthy postmenopausal women. Obstet Gynecol 1994;83:979-983. [Context Link] 
2. Robinson D, Friedman L, Marcus R, Tinklenberg J, Yesavage J. Estrogen replacement therapy and memory in older women. J Am Geriatr Soc 1994;42:919-922. Bibliographic Links | [Context Link]

3. Paganini-Hill A, Henderson VW. Estrogen deficiency and risk of Alzheimer's disease in women. Am J Epidemiol 1994;140:256-261. Bibliographic Links |[Context Link]

4. Morrison A, Resnick S, Corrada M, Zonderman A, Kawas C. A prospective study of estrogen replacement therapy and the risk of developing Alzheimer's disease in the Baltimore Longitudinal Study of Aging. Neurology 1996;46:A435. Bibliographic Links | [Context Link]

5. Tang M-X, Jacobs D, Stern Y, et al. Effect of oestrogen during menopause on risk and age at onset of Alzheimer's disease. Lancet 1996;348:429-432. [Context Link]

6. Schneider LS, Farlow MR, Henderson VW, Pogoda JM. Effects of estrogen replacement therapy on response to Tacrine in patients with Alzheimer's disease. Neurology 1996;46:1580-1584. Ovid Full Text | Bibliographic Links [Context Link]

7. Resnick SM, Metter EJ, Zonderman AB. Estrogen replacement therapy and longitudinal decline in visual memory: a possible protective effect? Neurology 1997;49:1491-1497. Ovid Full Text | Bibliographic Links |[Context Link]

8. Barrett-Connor E, Kritz-Silverstein D. Estrogen replacement therapy and cognitive function in older women. JAMA 1993;269:2637-2641. Bibliographic Links | [Context Link]

9. Rubin EH, Morris JC, Grant EA, Vendegna T. Very mild senile dementia of the Alzheimer type I. Clinical assessment. Arch Neurol 1989;46:379-382. Bibliographic Links |[Context Link]

10. Stern Y, Andrews H, Pittman J, et al. Diagnosis of dementia in a heterogeneous population. Development of a neuropsychological paradigm-based diagnosis of dementia and quantified correction for the effects of education. Arch Neurol 1992;49:453-460. Bibliographic Links | [Context Link]

11. Jacobs DM, Sano M, Dooneief G, Marder K, Bell KL, Stern Y. Neuropsychological detection and characterization of preclinical Alzheimer's disease. Neurology 1995;45:957-962. Ovid Full Text | Bibliographic Links |[Context Link]

12. Masur DM, Fuld PA, Blau AD, Crystal H, Aronson MK. Predicting development of dementia in the elderly with the Selective Reminding Test. J Clin Exp Neuropsychol 1990;12:529-538. Bibliographic Links | [Context Link]

13. Masur DM, Sliwinski M, Lipton RB, Blau AD, Crystal H. Neuropsychological prediction of dementia and absence of dementia in healthy elderly persons. Neurology 1994;44:1427-1432. Bibliographic Links | [Context Link]

14. Buschke H, Fuld PA. Evaluating storage, retention, and retrieval in disordered memory and learning. Neurology 1974;24:1019-1025. Bibliographic Links | [Context Link]

15. Wechsler D. Wechsler Adult Intelligence Scale-Revised. New York: The Psychological Corporation, 1981. [Context Link]

16. Kaplan E, Goodglass H, Weintraub S. Boston Naming Test. Philadelphia, PA: Lea \& Febiger, 1983. [Context Link] 
17. Mayeux R, Ottman R, Tang MX, et al. Genetic susceptibility and head injury as risk factors for Alzheimer's disease among community-dwelling elderly persons and their first-degree relatives. Ann Neurol 1993;33:494-501. [Context Link]

18. Fleiss JL. Statistical methods for rates and proportions. New York: John Wiley \& Sons Inc., 1981. [Context Link]

19. Sherwin BB. Sex hormones and psychological functioning in postmenopausal women. Exp Gerontol 1994;29:423-430. Bibliographic Links $\mid$ [Context Link]

20. Sherwin BB. Estrogenic effects on memory in women. Ann NY Acad Sci 1994;743:213-230. Bibliographic Links |[Context Link]

21. Gibbs RB. Estrogen and nerve growth factor-related systems in brain. Effects on basal forebrain cholinergic neurons and implications for learning and memory processes and aging. Ann NY Acad Sci 1994;743:165-196. [Context Link]

22. Gould E, Woolley CS, Frankfurt M, McEwen BS. Gonadal steroids regulate dendritic spine density in hippocampal pyramidal cells in adulthood. J Neurosci 1190;10:1286-1291. [Context Link]

23. Luine VN, Khylchevskaya RI, McEwen BS. Effect of gonadal steroids on activities of monoamine oxidase and choline acetylase in rat brain. Brain Res 1975;86:293-306. Bibliographic Links | [Context Link]

24. Miranda RC, Sohrabji F, Toran-Allerand CD. Presumptive estrogen target neurons express mRNAs for both the neurotrophins and neurotrophin receptors: a basis for potential developmental interactions of estrogen with the neurotrophins. Mol Cell Neurosci 1993;4:510-525. Bibliographic Links | [Context Link]

\section{IMAGE GALLERY}

Select All

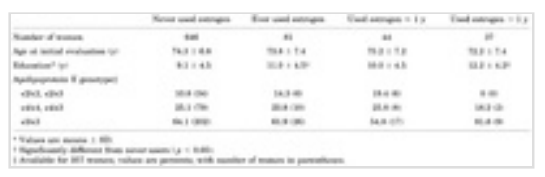

Table 1

Back to Top

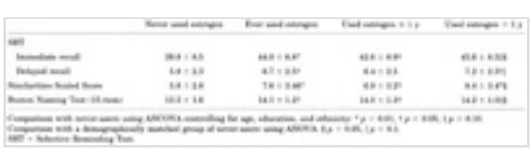

Table 2
Export Selected to PowerPoint

About Us Contact Us Privacy Policy Terms of Use

(C) 2020 Ovid Technologies, Inc. All rights reserved.

OvidUI_04.07.00.048, SourcelD a660381be3b297ad5e72f99d502151834ac9879d 